\section{Snaring quarks}

\author{
J.H. Mulvey
}

The Particle Hunters. By Yuval Ne'eman and Yoram Kirsh. Cambridge University Press:1986. Pp.272. Hbk£25, \$49.50; pbk £7.95, \$13.95.

As A concept the atom is more than 2,000 years old, but it is less than 200 years since Dalton's quantitative studies of chemical reactions gave it an observational basis. The original "particle hunter" was J.J. Thomson who discovered the first subatomic particle, the electron, in 1897. Soon after, in the years between 1910 and 1932 , the nature of the atomic nucleus was revealed largely by the work of Rutherford and his collaborators. Together with the accompanying development of quantum mechanics and special relativity, which revolutionized our understanding of the laws of physics, these discoveries underlie much of modern technology and our growing power to manipulate the molecules of life.

In their book, Ne'eman and Kirsh introduce the general reader to the microworld of particles. They start with Democritus and, passing by way of the gold-leaf electroscope, the conundrum of particlewave duality, the enigma of a left-right asymmetry in nature, and the invention of particle accelerators, which allowed physicists to become more like farmers than hunters, they arrive at the explosion of particle species found, mainly, in the late $1950 \mathrm{~s}$ and early $1960 \mathrm{~s}$. At the time, these observations seemed to confound all hope of rational explanation. Then, in 1964, Ne'eman and, independently, Gell Mann brought confusion to an end with their classification scheme based on the group SU(3), so performing for particle physics what Mendeleev had accomplished for atoms. This in turn soon led to the concept of quarks as constituents of all particles such as the proton and neutron.

The story is well told, apart from a few weak patches, and is enlivened by a sprinkling of personal reminiscences. Although their approach is non-mathematical, the authors do not condescend by evading some of the harder concepts.

The remaining 40 or so pages are not enough to do justice to all the triumphs which have followed, and the pace becomes quite breathless when telling of the recent discoveries of the $\mathrm{W}$ and $\mathrm{Z}$ bosons at CERN. But this book is a very valuable addition to the growing library bringing to the non-professional reader an appreciation of the ideas and methods used in the search for an understanding of the structure of matter.

J.H. Mulvey is a Senior Research Officer in the Nuclear Physics Laboratory, University of Oxford, Keble Road, Oxford OX1 3RH, UK.
Gödel: incomplete success

\section{David Miller}

Collected Works of Kurt Gödel. Volume I, Publications 1929-1936. Edited by Solomon Feferman et al. Oxford University Press: 1986. Pp. 474. £25, \$35.

IN HIS doctoral dissertation of 1929 , Kurt Gödel established the completeness of the usual formulations of elementary logic. In the following year he proved the incompleteness of every effective formulation of number theory. These two results, the second as devastating and unexpected as the first was satisfying, were announced to the mathematical public by the 24-yearold Gödel on successive days at the second Conference on Epistemology of the Exact Sciences (Tagung für Erklenntnislehre der exakten Wissenschaften) held in Königsberg in September 1930. There can be no doubt as to their cardinal importance in the history of modern logic.

The gist of Gödel's incompleteness theorem is this: there is no effective way of axiomatizing the set of true statements about the natural numbers, either in firstorder logic (which, by Gödel's completeness theorem, suffices for all correct deductions at that level) or in higher-order logic (type theory). That is, for any (consistent) axiomatic system $\mathbf{A}$, including any extension of Peano arithmetic (PA), there are infinitely many arithmetical truths not among its theorems. Gödel showed that one such truth is a statement $G$ that, within PA (or whatever axiomatic system $\mathbf{A}$ is under consideration), is provably equivalent to the assertion that " $G$ is not a theorem of $\mathbf{A}$ ". His demonstration relies on showing that, provided that the system of axioms is effectively given, the correctness of any arithmetical proof can be checked by arithmetical methods. For then if $\mathrm{G}$ is a theorem of $\mathbf{A}$ there is a proof $G$ of it, so that we can prove in $\mathbf{A}$ that " $G$ is a proof of G", thus proving in $\mathbf{A}$ that " $G$ is a theorem of A"; which is to say that we can prove not- $G$ in $\mathbf{A}$. Thus if $G$ is a theorem of $\mathbf{A}$ so also is not-G. So if $\mathbf{A}$ is consistent, $\mathrm{G}$ is not a theorem of $\mathbf{A}$. But this is what $\mathrm{G}$ says, so $\mathrm{G}$ is true.

Gödel's second incompleteness theorem (discovered also by von Neumann a few weeks after Königsberg) is obtained by showing that the above result - "if PA is consistent, $\mathrm{G}$ is not a theorem of PA" is itself a theorem of PA. For then if "PA is consistent" is a theorem of PA, so also is " $\mathrm{G}$ is not a theorem of PA"; that is, G is, which it is not. So "PA is consistent" is not a theorem of PA. Like the first incompleteness theorem this result applies to all effective extensions of PA. It rocked, but did not quite capsize, the hopes of Hilbert's school that it would be possible to show by proofs referring only to finite objects the consistency of the bulk of classical mathematics.

Gödel's published works will be issued as two volumes of his Collected Works, to be followed eventually by an unstated number of volumes containing unpublished manuscripts, lecture notes, and selections from his notebooks and correspondence. All items originally written in German will be reproduced with a translation on the facing page. This first volume contains Gödel's dissertation, and also a published version of it, slightly but tellingly different; his great paper on incompleteness; lecture notes on incompleteness (taken by Kleene and Rosser at Princeton in 1934); the text of his remarks at Königsberg; a brief note on what are today called speed-up theorems; two papers on the decision problem for elementary logic;

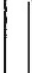

